\title{
Olfactory and Taste Dysfunction in Indian Population with COVID-19: A Study on 300 Patients
}

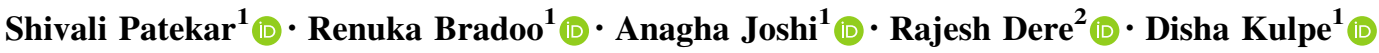

Received: 27 October 2021 / Accepted: 28 November 2021 / Published online: 10 January 2022

(C) Association of Otolaryngologists of India 2021

\begin{abstract}
The novel coronavirus disease 2019 is an ongoing pandemic and a global health emergency. Since the main portal of entry for the virus is the nose, olfactory and taste dysfunction have gained attention as important symptoms in COVID-19. The primary objectives are to assess the prevalence of olfactory and taste dysfunction in patients affected with COVID-19. And to determine whether anosmia and ageusia are early predictors of COVID19. A prospective study was conducted on 300 patients who were laboratory diagnosed cases of COVID-19 admitted in tertiary care centre and a covid care centre. A written informed consent was taken. All patients underwent olfactory and taste examination and the details were noted in a case-record proforma. The prevalence of olfactory dysfunction in COVID-19 patients was $21 \%$ and that of taste dysfunction was $26.6 \%$. Majority of the affected individuals were males and mainly of the younger age group. These symptoms lasted for a duration of 7-9 days and $97 \%$ of the patients showed complete recovery. Acute sudden onset olfactory and taste dysfunction seems to be a useful indicator for early diagnosis of patients thus helping in early isolation, timely management of the illness and controlling the spread of the disease.
\end{abstract}

Shivali Patekar

shivalip1990@gmail.com

1 Department of ENT and Head and Neck Surgery, Lokmanya Tilak Municipal Medical College and General Hospital, Sion, Mumbai, Maharashtra, India

2 Department of Forensic Medicine and Toxicology, Lokmanya Tilak Municipal Medical College and General Hospital, Sion, Mumbai, Maharashtra, India
Keywords COVID-19 · Anosmia $\cdot$ Loss of taste . Olfactory dysfunction

\section{Introduction}

The coronavirus disease 2019 (COVID-19) was first identified in December 2019 in Wuhan, China and was subsequently found to be caused by a novel coronavirus, now referred to as Severe Acute Respiratory Syndrome Coronavirus 2 (SARS-CoV-2). Since then it has spread rapidly across the world and was declared by the World Health Organization as a pandemic and global health emergency on March 11, 2020 [1]. Infection by SARSCoV-2 may occur via inhaled particles such as aerosols (less than $5 \mu \mathrm{m}$ in size; capable of staying suspended in the air for long periods of time and easily inhaled into the lungs and distal alveoli) or droplets (measuring greater than $20 \mu \mathrm{m}$ in size; mostly deposited in the nasal cavity), or by direct inoculation of the respiratory epithelium (ie, touching a surface with live virus and then touching one's face). Since the main portal of entry of the virus is the nose, it is possible that the sinonasal cavities may be important sites for initial infection by SARS-CoV-2 [2]. Constitutional symptoms, such as fever, cough, fatigue, shortness of breath, and myalgia, are commonly reported. However, olfactory dysfunction (hyposmia or anosmia) and loss of taste have gained attention as an important symptom in COVID-19 [2]. Thus, the study was conducted to assess the prevalence, time relation with respect to the COVID RT PCR testing date and recovery pattern of olfactory and taste dysfunction in COVID-19 positive patients.

The aim of the study were to assess the prevalence of olfactory and taste dysfunction in COVID 19 positive 
patients and to determine whether loss of smell and loss of taste are early predictive symptoms of COVID- 19.

\section{Methodology}

A prospective study was conducted on 300 patients who were laboratory diagnosed cases of COVID-19. It was a multi-institutional study conducted in a tertiary care centre and a covid care centre.

\section{Inclusion Criteria}

- Age more than 18 years

- Laboratory confirmed COVID positive patients who were able to answer the questionnaire.

\section{Exclusion Criteria}

- Patients who are laboratory confirmed COVID positive but critically ill.

- Patients with pre-existing Anosmia and Loss of taste.

- Pregnant women

The study was conducted with the approval of the ethics committee of the hospital. All the patients were provided detailed information of the study and a written informed consent was taken for the same. Patients who consented were included in the study and were followed up for three months. Along with demographic data, history regarding Co- morbidities, ENT symptomatology and other COVID related symptoms were noted. Olfactory examination was done on all patients using Coffee, garlic, lemon and rose. Gustatory examination was done using sugar (sweet), salt, coffee (bitter) and lemon (sour). If the patients had olfactory and/or taste dysfunction, detailed history regarding the onset with respect to the COVID testing day, duration, severity, its association with other symptoms and its recovery whether complete or partial were noted in a case record proforma.

\section{Results}

Of the 300 patients in the study, males were more affected than females (Table 1). The mean age was found to be 45.90(16.12) years (Table 2).

The participants characteristics with respect to ENT symptoms and general Co-morbidities has been listed in Table 3

The most prevalent co-morbidities in these patients were Diabetes Mellitus followed by Hypertension. Most common ENT symptoms seen in these patients were loss of taste (ageusia) and anosmia followed by rhinitis, sorethroat, dry cough etc. (Table 3). Out of 300 patients 106 had fever which was $35.4 \%$. Pneumonia was seen in $4.4 \%$ patients.

\section{Olfactory Dysfunction outcome}

Amongst 300 patients,63(21\%) patients had Olfactory dysfunction. $70 \%$ of the patients affected were males and mainly of the younger age group. 47 out of $63(74.6 \%)$ patients had complete anosmia whereas $16(25.4 \%)$ patients had hyposmia. 44 out of $63(69.8 \%)$ patients presented with olfactory dysfunction before the day of COVID testing, 8 patients showed olfactory dysfunction at the same day of testing whereas 11 patients showed symptom after the day of COVID testing (Table 5). 24 out of 63(38.7\%) patients had anosmia lasting for 4-7 days and 10(16.1\%) patients has anosmia for less than 3 days. In $36 \%$ of the patients the duration was 8-14 days whereas $10 \%$ of the patients had anosmia for more than 14 days. The mean duration of loss of smell in affected patients was 7.90 days (Table 4). 54\% of the patients recovered within 7 days of onset of anosmia. $96.8 \%$ of the patients showed complete recovery from anosmia.

\section{Taste Dysfunction outcomes}

In our study, 80 of $300(26.6 \%)$ patients had loss of taste. $73 \%$ of patients affected were males mainly of the age group of 20-30 years. 53 out of 80 patients presented with loss of taste before the day of COVID testing, 11 patients had loss of taste from the day of testing and 17 patients had loss of taste after the day of tasting(Table 5 ). $41.3 \%$ of the

Table 1 Distribution of study subjects according to the gender $(\mathrm{N}=300)$

\begin{tabular}{lll}
\hline Gender & No & Percent \\
\hline Male & 194 & 64.7 \\
Female & 106 & 35.3 \\
\hline
\end{tabular}


Table 2 Distribution of study subjects according to the age group $(\mathrm{N}=300)$

\begin{tabular}{llr}
\hline Age (in years) & No & Percent \\
\hline$\leq 20$ & 7 & 2.3 \\
$21-30$ & 64 & 21.3 \\
$31-40$ & 45 & 15.0 \\
$41-50$ & 59 & 19.7 \\
$51-60$ & 61 & 20.3 \\
$61-70$ & 46 & 15.3 \\
$71-80$ & 14 & 4.7 \\
$>80$ & 4 & 1.3 \\
Mean (SD) & $45.90(16.12)$ & \\
Range & $16-90$ & \\
\hline
\end{tabular}

Table 3 Distribution of study subjects according to the ENT symptoms ( $\mathrm{N}=300)$

\begin{tabular}{lll}
\hline Symptoms & No & Percent \\
\hline Loss of taste & 80 & 26.6 \\
Anosmia & 63 & 21 \\
Rhinitis & 37 & 12.3 \\
Fever & 106 & 35.33 \\
Sore throat & 26 & 8.7 \\
Cough & 16 & 5.3 \\
Postnasal drip & 1 & 0.3 \\
Nasal blockade & 3 & 1.0 \\
Breathlessness & 2 & 0.7 \\
Hoarseness & 2 & 0.7 \\
Hearing loss & 1 & 0.3 \\
Comorbidities & & 13.3 \\
Diabetes mellitus & 40 & 11.3 \\
Hypertension & 34 & 0.7 \\
Thyroid disorder & 2 & 0.7 \\
Schizophrenia & 2 & 0.7 \\
Ischemic heart Disease & 1 & 0.3 \\
Chronic heart Disease & 1 & 0.3 \\
Asthma & 1 &
\end{tabular}

Table 4 Distribution of study subjects according to the duration

\begin{tabular}{lll}
\hline Duration & Loss of smell $(\%)$ & Loss of taste $(\%)$ \\
\hline $1-3$ & $10(16.1)$ & $12(15.0)$ \\
$4-7$ & $24(38.7)$ & $33(41.3)$ \\
$8-14$ & $22(35.5)$ & $25(31.3)$ \\
$>14$ & $6(9.7)$ & $10(12.5)$ \\
Mean (SD) & $7.90(4.74)$ & $8.05(5.37)$ \\
\hline
\end{tabular}


Table 5 Distribution of study subjects according to the onset $(\mathrm{N}=300)$

\begin{tabular}{|c|c|c|}
\hline Onset & Loss of smell $(\%)$ & Loss of taste $(\%)$ \\
\hline \multicolumn{3}{|c|}{ Days before COVID testing } \\
\hline 15 & & $1(1.9)$ \\
\hline 10 & $1(2.3)$ & $1(1.9)$ \\
\hline 8 & $1(2.3)$ & $3(5.7)$ \\
\hline 7 & $5(11.4)$ & $4(7.6)$ \\
\hline 6 & $1(2.3)$ & $1(1.9)$ \\
\hline 5 & $4(9.1)$ & $4(7.6)$ \\
\hline 4 & $4(9.1)$ & $4(7.6)$ \\
\hline 3 & $7(15.9)$ & $6(11.5)$ \\
\hline 2 & $17(38.6)$ & $20(38.4)$ \\
\hline 1 & $4(9.1)$ & $8(15.3)$ \\
\hline Total before & $44(69.8)$ & $52(65)$ \\
\hline On the day of testing & $8(12.7)$ & $11(13.6)$ \\
\hline \multicolumn{3}{|c|}{ Days after COVID testing } \\
\hline 1 & $2(18.2)$ & 3 (17.6) \\
\hline 2 & $6(54.5)$ & $8(47.1)$ \\
\hline 3 & $1(9.1)$ & $3(3.7)$ \\
\hline 4 & $1(9.1)$ & \\
\hline 7 & & $2(2.5)$ \\
\hline 10 & $1(9.1)$ & $1(1.2)$ \\
\hline Total after & $11(17.5)$ & $17(21.0)$ \\
\hline Total & $63(21.0)$ & $80(26.6)$ \\
\hline
\end{tabular}

patients had loss of taste for around 4-7 days whereas $15 \%$ had loss of taste for less than 3 days. In $31.3 \%$ of the patients loss of taste lasted for around 8-14 days while $12.5 \%$ of the patients had loss of taste for more than 14 days (Table 4 ). The mean duration of loss of taste in the affected individuals was 8.05 days. $97.5 \%$ of the patients showed complete recovery of taste.

\section{Discussion}

COVID-19 outbreak is a pandemic affecting people all over the world, not just disrupting the global situation but also destabilizing world economy. During the early days of the pandemic, symptoms such as fever, cough, shortness of breath were mainly encountered with the disease. Later on, olfactory dysfunction and loss of taste were identified as important early symptoms of COVID 19.

Various theories regarding pathophysiology of anosmia in COVID-19 positive patients have been studied recently. A study by Sungnak et al. [3] suggested that nasal epithelial cells show a high angiotensin converting enzyme 2 (ACE2) expression in SARS-CoV-2 infection, and thus this may allow the viral entry. The binding of the virus to
ACE 2 receptors causes degeneration of the epithelial cells of the nasal mucosa and subsequent inflammation and damage to the neural receptors responsible for olfaction. It may have significance in identifying asymptomatic carriers or those with mild symptoms that would otherwise not raise suspicion for COVID-19 [1]. Another study done by David H. Brann et al. on pathophysiology of acute loss of smell reported in patients with COVID-19 suggests four possible mechanisms. First, local infection of support and vascular cells in the nose and olfactory bulb could cause significant inflammatory responses (including cytokine release) whose effects could block effective olfactory conduction or alter the function of olfactory sensory neuroepithelium (OSN). Second, damage to support cells (which are responsible for local water and ion balance) could indirectly influence signaling from OSNs to the brain. Third, damage to Sustentacular cells and Bowman gland cells in mouse models can lead to OSN death, which in turn could affect smell perception. Lastly, vascular damage could lead to hypoperfusion and inflammation leading to changes in Olfactory bulb function [4].

The prevalence of olfactory dysfunction (hyposmia and anosmia) in our study was $21 \%$. A study conducted by Mishra $\mathrm{P}$ et al. in Indian population, showed the prevalence 
of $14 \%$ [5]. One of the studies conducted by Lechien et al. showed the prevalence of olfactory dysfunction in European population as high as $85.6 \%$ [6] whereas in another study conducted by Klopfenstein it was $47 \%$ [7]. The prevalence of olfactory dysfunction in Indian population seems to be lesser as compared to European population. The reason for such a difference in anosmia prevalence rates between European and Asian population is still unclear. These patients were treated with decongestants and steam inhalation.

In our study $26.6 \%$ of the patients reported taste dysfunction. Ageusia may be a secondary result of olfactory dysfunction. The angiotensin-converting enzyme 2 receptor, which is the main host cell receptor of SARS-CoV-2 for binding and penetrating cells, is widely expressed on epithelial cells of the oral mucosa. Damage to the mucosal epithelial cells of the oral cavity may explain loss of taste observed in the early stage of COVID-19 [8].

In our study, the prevalence of taste dysfunction was more than the prevalence of olfactory dysfunction. $70 \%$ of the patients affected with olfactory and $73 \%$ of the patients affected with taste disturbances were males. Though middle-aged patients were more affected with COVID-19, taste and olfactory dysfunction were mainly seen in younger age groups (20-30). A study conducted by A Izquierdo-Domínguez et al. found similar results i.e. loss of taste was more affected than loss of smell in patients with COVID-19. It also stated that younger age group was more affected with loss of taste and smell than the older age group [9].

Around $70 \%$ of the patients had both loss of smell and loss of taste before testing of COVID-19 suggesting that both the symptoms are having higher positive predictive value for COVID-19 i.e. if both loss of smell and loss of taste are present in any patient, he is more likely to be affected with COVID-19. These symptoms lasted for around 7-9 days and showed complete recovery in $97 \%$ of the patients. A study conducted by Lechien et.al also showed olfactory and taste dysfunction lasting for around 8 days with complete resolution in $67.8 \%$ of the patients [6].

In our study of 300 selected patients the ones which could not answer the questionnaire were excluded hence the incidence of pneumonia seen was less. 13 of our patients had pneumonia.

\section{Conclusion}

The novel COVID-19 started as an uncertain, serious and confusing illness. It later became a pandemic with different mutations and clinical presentations. The sudden onset anosmia or ageusia are recognized by the international scientific community as important symptoms of the
COVID-19 infection. Our study showed prevalence of anosmia in Indian population to be $21 \%$ and of ageusia to be $26.6 \%$. Based on our results, it seems that patients with acute onset olfactory and taste dysfunction are more likely to have COVID-19. As early diagnosis is important for the control of COVID-19, recognition of early signs such as anosmia or ageusia might be a useful indicator for the diagnosis COVID-19 and isolation of the patients.

Author Contribution Dr. Renuka Bradoo and Dr. Anagha Joshi had peer reviewed our article and helped in guiding, analysis and and proof-reading the data. Dr. Shivali Patekar had contributed with the idea of writing the article, interpretation of data and review of literature. Dr. Rajesh Dere and Dr Disha Kulpe helped in collecting data.

Funding The author(s) received no financial support for the research, authorship and/or publication of this article.

\section{Declarations}

Conflict of interest The author(s) declared no potential conflicts of interest with respect to the research, authorship, and/or publication of this article.

Informed consent Written, informed and valid consent taken from the patients.

\section{References}

1. Speth MM, Singer-Cornelius T, Oberle M, Gengler I, Brockmeier SJ, Sedaghat AR (2020) Olfactory dysfunction and sinonasal symptomatology in COVID-19: Prevalence, severity, timing, and associated characteristics. Otolaryngol-Head Neck Surg 163(1):114-120

2. Gengler I, Wang JC, Speth MM, Sedaghat AR (2020) Sinonasal pathophysiology of SARS-CoV-2 and COVID-19: A systematic review of the current evidence Level of Evidence: 2a. Laryngoscope Investig Otolaryngol 5:354-359

3. Sungnak W, Huang N, Bécavin C, Berg M, Network HLB (2020) SARS-CoV-2 entry genes are most highly expressed in nasal goblet and ciliated cells within human airways. Nat Med 26(5):681-7. Available from: http://arxiv.org/abs/2003.06122

4. Brann DH, Tsukahara T, Weinreb C, Lipovsek M, Van den Berge K, Gong B, et al. (2020) Non-neuronal expression of SARS-CoV-2 entry genes in the olfactory system suggests mechanisms underlying COVID-19-associated anosmia. Sci Adv 6. Available from: http://advances.sciencemag.org/

5. Mishra P, Gowda V, Dixit S, Kaushik M (2020) Prevalence of new onset anosmia in COVID-19 patients: Is the trend different between European and Indian population? Indian J Otolaryngol Head Neck Surg 72(4):484-487

6. Lechien JR, Chiesa-Estomba CM, De Siati DR, Horoi M, Le Bon SD, Rodriguez A et al (2020) Olfactory and gustatory dysfunctions as a clinical presentation of mild-to-moderate forms of the coronavirus disease (COVID-19): a multicenter European study. Eur Arch Oto-Rhino-Laryngology 277(8):2251-2261. https://doi.org/10.1007/s00405-020-05965-1

7. Klopfenstein T, Kadiane-Oussou NJ, Toko L, Royer PY, Lepiller $\mathrm{Q}$, Gendrin V et al (2020) Features of anosmia in COVID-19. Med 
Mal

Infect

50(5):436-439.

https://doi.org/10.1016/j.medmal.2020.04.006

8. Lee Y, Min P, Lee S, Kim S-W (2020) Prevalence and duration of acute loss of smell or taste in COVID-19 patients. J Korean Med Sci 35(18):e174.

https://doi.org/10.3346/jkms.2020.35.e174eISSN1598-

6357.pISSN1011-8934

9. Izquierdo-Domínguez A, Rojas-Lechuga MJ, Chiesa-Estomba C, Calvo-Henríquez C, Ninchritz-Becerra E, Soriano-Reixach M, et al. (2020) Smell and taste dysfunction in covid-19 is associated with younger age in ambulatory settings: A multicenter crosssectional study. J Investig Allergol Clin Immunol 30(5):346-57. Available from: https://pubmed.ncbi.nlm.nih.gov/32554337/

Publisher's Note Springer Nature remains neutral with regard to jurisdictional claims in published maps and institutional affiliations. 